\title{
Invasive micropapillary carcinomas of the ampullo-pancreatobiliary region and their association with tumor-infiltrating neutrophils
}

\author{
Said Khayyata, Olca Basturk and Nazmi V Adsay \\ Department of Pathology, The Karmanos Cancer Institute and Harper University Hospital, Wayne State \\ University, Detroit, MI, USA
}

\begin{abstract}
Invasive micropapillary carcinoma, originally described as a distinctive type of invasive carcinoma in the breast, is being increasingly recognized as a separate entity in many other organs; however, it has not yet been documented in the pancreas or periampullary region. In this study, 313 pancreatic and 73 periampullary carcinomas were reviewed to investigate the micropapillary pattern in this location. Eight periampullary and eight pancreatic cases $(4 \%)$ were composed at least focally $(>20 \%)$ of invasive micropapillary carcinoma. The patients were 10 males and six females, mean age 69 years. The mean tumor size was $3.2 \mathrm{~cm}$. Lymph node metastasis was detected in 11/15 cases. The median survival was 8 months (all were resected). Invasive micropapillary carcinoma was characterized by small, closely packed micropapillary clusters (without fibrovascular cores) lying within clefts. The cells had moderate degree of nuclear atypia. In nine cases, there was abundant inflammation composed of neutrophils concentrating around the tumor cells, both intraepithelial ('cannibalism') and stromal. Molecules implicated in abnormalities of tumor cell-stroma adhesion, galectin-3 and E-cadherin were expressed in the cytoplasm of $11 / 11$ and 9/11 cases, respectively. Reversal of cell polarity was observed by MUC 1 in all 11 cases tested, which showed labeling in the stroma-facing surfaces of the micropapillary clusters, also confirming that the clefts are not merely a processing artifact, but indeed a true biologic alteration. In conclusion, invasive micropapillary carcinoma constitutes $4 \%$ of carcinomas in the pancreatic/periampullary region and is commonly associated with abundant neutrophilic infiltrates. Invasive miropapillary carcinoma appears to be more common in periampullary than in pancreatic invasive micropapillary carcinoma would qualify as poorly differentiated both based on pattern and the median survival (8 months).

Modern Pathology (2005) 18, 1504-1511. doi:10.1038/modpathol.3800460; published online 8 July 2005
\end{abstract}

Keywords: micropapillary; pancreatobiliary; ampullary

Originally described in the breast, invasive micropapillary carcinoma is being increasingly recognized as a separate entity in other organs including the urinary bladder, lung, major salivary glands and ovary. ${ }^{1-6}$ It is characterized by small neoplastic cell clusters, surrounded by a peculiar cleft. ${ }^{7}$ Invasive micropapillary carcinoma may manifest as a focal pattern, or may constitute the majority of a tumor. In the organs in which it is best characterized, invasive micropapillary carcinoma has been shown to have a highly infiltrative nature, often presenting at advanced stages, and showing high degrees of lymphotrophism. ${ }^{8,9}$ To our knowledge, invasive

Correspondence: Dr NV Adsay, MD, Department of Pathology, The Karmanos Cancer Institute and Harper University Hospital, Wayne State University, Detroit, MI 48201, USA.

E-mail: adsayv@med.wayne.edu

Received 28 March 2005; revised 25 May 2005; accepted 28 May 2005; published online 8 July 2005 micropapillary carcinoma has not yet been reported in the ampullo-pancreatobiliary region.

In this study, carcinomas of this region resected at the authors' institution were reviewed to identify those with micropapillary pattern, determine their relative frequency, and document their clinicopathologic features.

\section{Materials and methods}

This study was conducted in accordance with a protocol approved by the institutional review board of the Wayne State University.

In all, 313 pancreatic and 73 periampullary carcinomas that underwent resection in the authors' institution were reviewed to investigate the presence of micropapillary pattern. The extent of the micropapillary component in the primary tumor was arbitrarily classified as focal $(20-50 \%$ of the 
tumor), predominant $(>50 \%)$ or diffuse $(>80 \%)$. Those with less than $20 \%$ were not included into the study. Also, carcinomas in which angioinvasion exhibited a micropapillary pattern were also excluded, unless the stromal component of the tumor also exhibited the characteristic micropapillary carcinoma.

Pathology material and the reports of the cases were analyzed for demographic data (age and gender), pathologic staging parameters (lymph node and tumor size), other pathologic characteristics (vascular and perineurial invasion) and clinical outcome. Follow-up information was obtained from the patient's charts, by contacting the primary physicians, or through the Surveillance and Epidemiology End Results (SEER) database. The findings were compared with those of conventional carcinomas.

Immunohistochemical stains were performed to determine the nature of the clefts that characterize the micropapillary pattern, and to verify that they are not processing artifacts. CD31, a marker of endothelial cells, was used to investigate what percentage, if any, of these spaces represented vascular spaces. The expression of E-cadherin and galectin-3, which have been implicated in the abnormalities of tumor cell host-stroma interaction, and which in turn may contribute to the detachment of cells from the stroma, were also analyzed. MUC 1, the expression pattern of which was recently found to confirm the reversal of cell polarity characteristic of micropapillary pattern was also tested. ${ }^{7,10}$ Immunohistochemical stains were performed using the avidin-biotin peroxidase complex method. Primary and secondary antibodies and the detection kit were purchased from commercial laboratories.

The specifics of the antibodies used are as follows: CD31 (clone JC/70A, Ventana, Tucson, AZ, USA), MUC 1 (clone Ma695, Novocastra, Newcastle, UK), E-cadherin (clone ECH-6, Ventana, Tucson, AZ, USA), and galectin-3 (Novocastra, Newcastle, UK), with the dilutions of: predilute, 1:100, predilute and 1:100 in mild CC1 or EDTA, citrate, EDTA or CC1 and citrate, respectively. Staining for CD31 and Ecadherin was run on the Ventana Automated system. The CD31, MUC 1, E-cadherin and galectin were incubated for $16 \mathrm{~min}, 2 \mathrm{~h}, 20 \mathrm{~min}$ and $2 \mathrm{~h}$, respectively.

Tonsil tissue was used as the control for galectin3 , breast cancer tissue for MUC 1 and galectin-3, and lung cancer tissue for E-cadherin.

Tissue blocks were available from 11 out of the 16 cases for immunohistochemistry.

Ultrastructural examination was performed on tissue originally placed in $10 \%$ formaldehyde and later transferred to $2.5 \%$ glutaraldehyde. The samples were postfixed with osmium tetraoxide, processed routinely, stained with lead citrate and examined under transmission electron microscopy.

\section{Results}

\section{The Frequency of Micropapillary Pattern}

Micropapillary pattern was identifiable in ampullopancreatobiliary carcinomas, at least focally, in $4 \%$ of the cases (16/386). The frequency of invasive micropapillary carcinoma appeared to be higher in periampullary $(11 \%)$ than in primary pancreatic carcinomas $(3 \%)$. Tumors composed of a diffuse MP pattern $(>80 \%)$, however, were exceedingly uncommon: $3 \%$ of periampullary and $1 \%$ of pancreatic carcinomas (two cases each). Six periampullary and six pancreatic cases were composed at least predominantly $(>50 \%)$ of micropapillary carcinoma. Another two periampullary and two pancreatic cases were composed only focally $(20-50 \%)$ of micropapillary carcinoma.

Of note, in the same database there was an example of metastatic urothelial carcinoma in the pancreas composed entirely of micropapillary pattern.

\section{Clinical Findings}

The 16 patients ranged in age from 41 to 80 years (mean 69 years) as opposed to the mean age of 63 in ordinary ductal carcinomas of the pancreas in the same database $(P=0.06)$. In all, 10 patients were male and six were female (62\% male) (vs $49 \%$ male in ordinary carcinomas). ${ }^{11}$

Clinical findings are summarized in Tables 1 and 2.

Table 1 Pancreatic tumors with micropapillary component

\begin{tabular}{llccccc}
\hline $\begin{array}{l}\text { Case } \\
\#\end{array}$ & $\begin{array}{l}\text { Age/ } \\
\text { sex }\end{array}$ & $\begin{array}{c}\text { Percentage of } \\
\text { micropapillary } \\
\text { component }\end{array}$ & $\begin{array}{c}\text { Tumor } \\
\text { size }\end{array}$ & $\begin{array}{c}\text { Lymph } \\
\text { node }\end{array}$ & $\begin{array}{c}\text { Survival } \\
\text { (months) }\end{array}$ & Status \\
\hline 1 & $80 / \mathrm{F}$ & $>80$ & 4.5 & $3 / 20$ & 2 & Dead \\
2 & $60 / \mathrm{F}$ & $>80$ & 3 & $0 / 17$ & 4 & Alive \\
3 & $79 / \mathrm{M}$ & $>50$ & 3 & $3 / 17$ & 7 & Dead \\
4 & $70 / \mathrm{F}$ & $>50$ & 2 & $8 / 8$ & 13 & Dead \\
5 & $77 / \mathrm{M}$ & $>50$ & 4 & $5 / 9$ & 15 & Alive \\
6 & $62 / \mathrm{M}$ & $>50$ & $\mathrm{~N} / \mathrm{A}$ & $\mathrm{N} / \mathrm{A}$ & 5 & Dead \\
7 & $73 / \mathrm{F}$ & $20-50$ & 3.5 & $3 / 7$ & 8 & Dead \\
8 & $69 / \mathrm{F}$ & $20-50$ & 4.5 & $1 / 1$ & 4 & Dead
\end{tabular}

$\mathrm{N} / \mathrm{A}=$ not applicable; $\mathrm{M}=$ male; $\mathrm{F}=$ female

Table 2 Periampullary tumors with micropapillary component

\begin{tabular}{llccccc}
\hline $\begin{array}{l}\text { Case } \\
\#\end{array}$ & $\begin{array}{l}\text { Age/ } \\
\text { sex }\end{array}$ & $\begin{array}{c}\text { Percentage of } \\
\text { micropapillary } \\
\text { component }\end{array}$ & $\begin{array}{c}\text { Tumor } \\
\text { size }\end{array}$ & $\begin{array}{c}\text { Lymph } \\
\text { node }\end{array}$ & $\begin{array}{c}\text { Survival } \\
\text { (months) }\end{array}$ & Status \\
\hline 1 & $79 / \mathrm{M}$ & $>80$ & 2.2 & $19 / 32$ & N/A & N/A \\
2 & $71 / \mathrm{M}$ & $>80$ & $\mathrm{~N} / \mathrm{A}$ & $0 / 4$ & 10 & Dead \\
3 & $41 / \mathrm{M}$ & $>50$ & 3.5 & $2 / 3$ & 6 & Dead \\
4 & $71 / \mathrm{M}$ & $>50$ & 1.5 & $2 / 9$ & 9 & Dead \\
5 & $62 / \mathrm{M}$ & $>50$ & 2 & $0 / 3$ & 25 & Dead \\
6 & $67 / \mathrm{M}$ & $>50$ & 3.5 & $1 / 1$ & 8 & Dead \\
7 & $76 / \mathrm{F}$ & $20-50$ & 2.8 & $4 / 17$ & N/A & N/A \\
8 & $61 / \mathrm{M}$ & $20-50$ & 2.5 & $0 / 9$ & 70 & Dead \\
\hline
\end{tabular}

$\mathrm{N} / \mathrm{A}=$ not applicable; $\mathrm{M}=$ male $; \mathrm{F}=$ female. 


\section{Pathologic Findings}

All pancreatic micropapillary carcinomas arose from the head of the pancreas.

The size of the primary tumors ranged from 1.5 to $4.5 \mathrm{~cm}$ (mean $3.2 \mathrm{~cm}$; median $3.0 \mathrm{~cm}$ ), and was similar to the mean and median of $3.0 \mathrm{~cm}$ for ordinary ductal carcinoma in the same database $(P=0.698)$, Tables 1 and 2 . The primary tumors were generally described as ill defined, with bright yellow solid cut surfaces.

Microscopically, invasive micropapillary carcinoma were characterized by small, round to ovoid micropapillary clusters (without fibrovascular cores), which were lying within clefts (Figures 1 and 2). In most areas, the micropapillary units were closely packed, with much less intervening stroma than is typically seen in carcinomas of this region. The cells had a fair amount of acidophilic

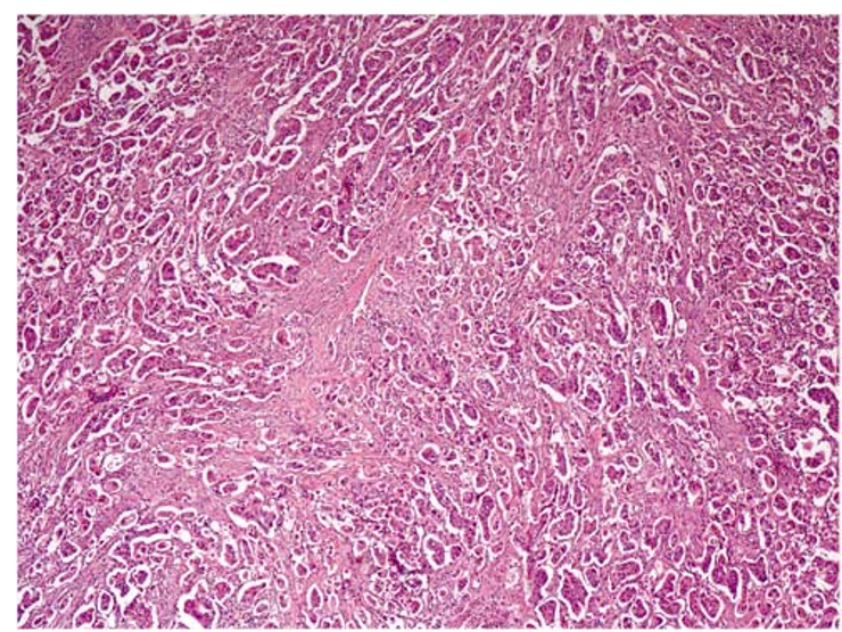

Figure 1 Invasive micropapillary carcinoma, low-power view.

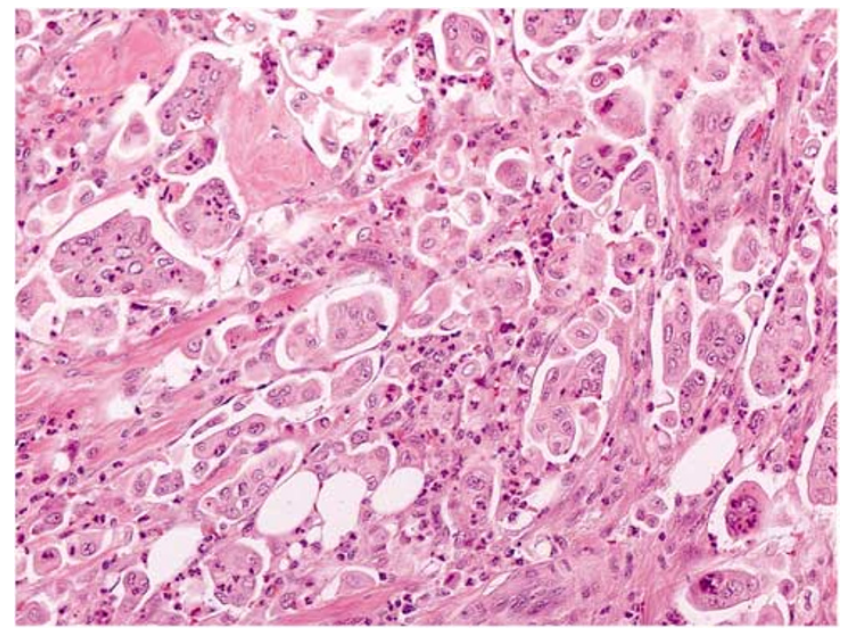

Figure 2 Invasive micropapillary carcinoma, classical features: ovoid clusters within clefts and moderate nuclear atypia. Stroma is less abundant than ordinary carcinoma. cytoplasm, and moderate degree of nuclear atypia. In nine cases, there was abundant inflammation composed of neutrophils, which were both intraepithelial, with focal microabscesses formation, and stromal (Figure 3). Neutrophils were much less prominent in the three cases in which MP component was less abundant. The concentration of the neutrophils in and around the tumor cells, along with the lack of any specific history or pathologic evidence of prior instrumentation, indicated that the presence of neutrophils was a primary process rather than secondary to instrumentation. No foreign-body giant cell reaction, suture granulomas, chronic inflammation, granulation tissue or scarring were identified in the vicinity of the tumor. Pathologic materials were available from metastatic invasive micropapillary carcinoma to lymph nodes in two cases; the metastatic foci exhibited the same micropapillary features in both cases, including the tumor-infiltrating neutrophils. In some areas, micropapillary clusters showed giant cell-like appearance and were associated with hypercellular stroma, with an overall appearance resembling osteoclastic giant cell carcinoma (Figure 4).

The three cases in which focal angioinvasion exhibited a micropapillary pattern were excluded from the study. This finding was focal, constituting less than $5 \%$ of the tumor, and was predominantly noted in the submucosal vasculature of the duodenum or ampulla (Figure 5).

\section{Immunohistochemical Findings}

MUC 1 immunostaining was predominantly in the stroma-facing surface of the cell clusters in all the 11 cases (Figure 6). In accordance with our recent study, MUC 1 staining was present exclusively on the stroma-facing surface of the micropapillary clusters. ${ }^{10}$

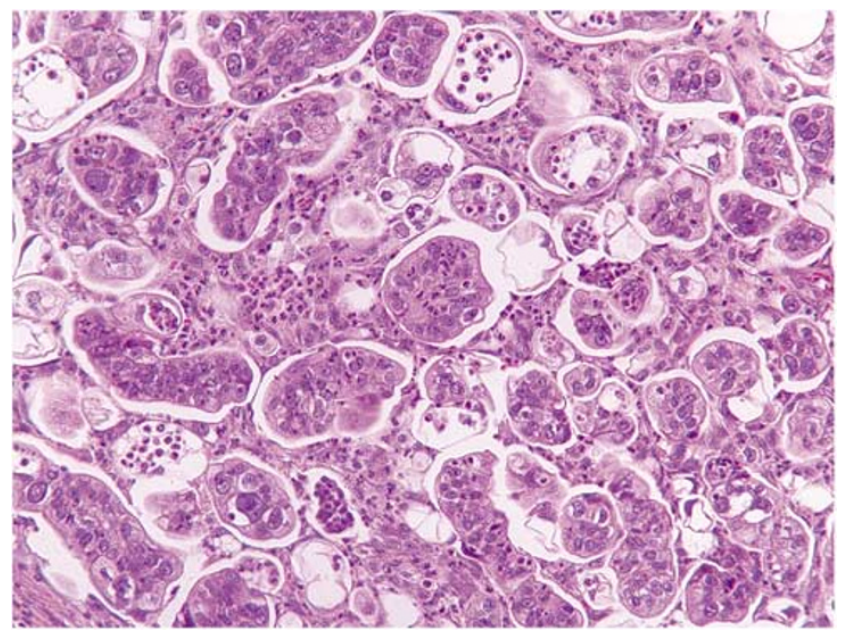

Figure 3 Invasive micropapillary carcinoma with intraepithelial and stromal tumor-infiltrating neutrophils. This could not be attributed to previous biopsy. 


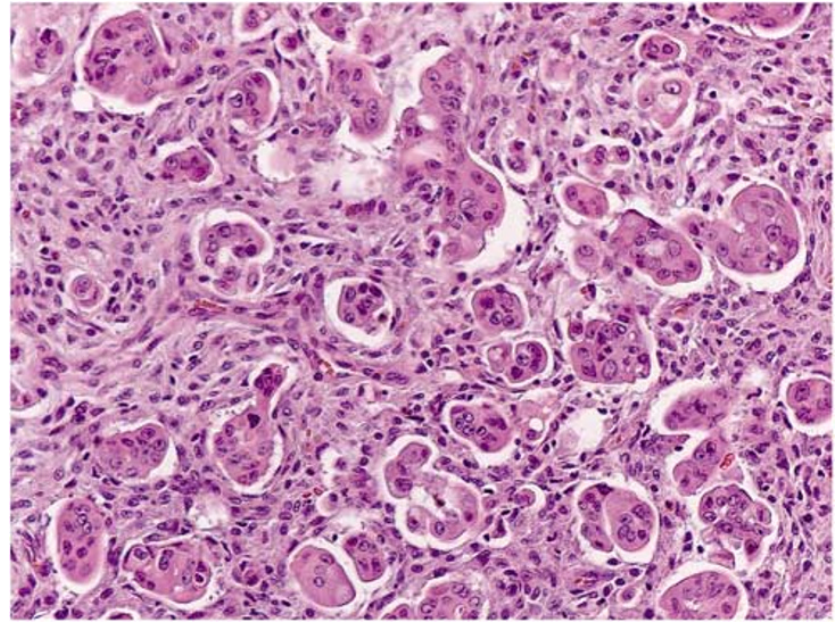

Figure 4 Invasive micropapillary carcinoma with giant cell-like appearance and hypercellular stroma, resembling osteoclastic giant cell carcinoma.

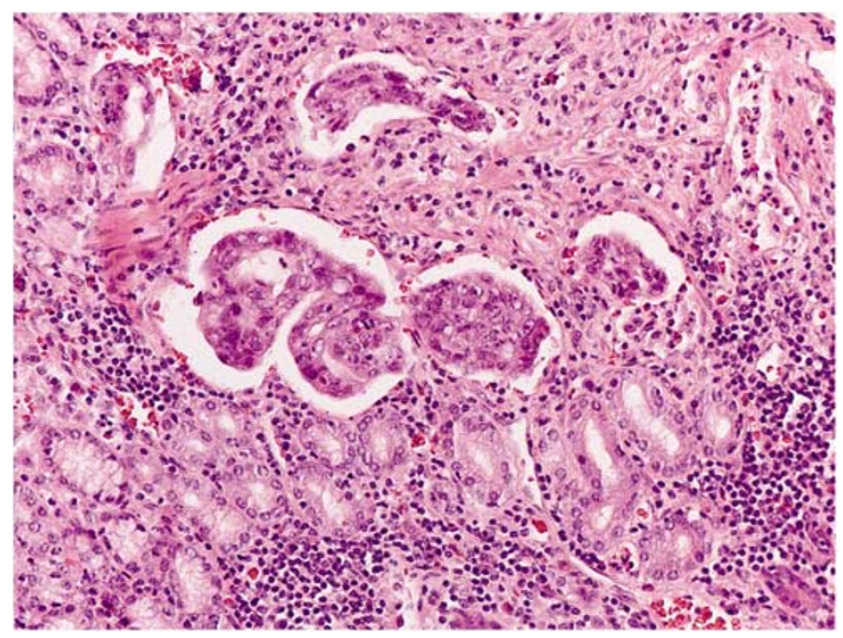

Figure 5 Submucosal angioinvasion with MP-like appearance not regarded as invasive micropapillary carcinoma IMPCa.
E-cadherin expression was detected in nine of 11 cases. In invasive micropapillary carcinoma areas, the labeling was predominantly cytoplasmic, rather than membranous as seen in normal ductal elements or in tubular components of the carcinomas (Figure 7). Cytoplasmic expression of E-cadherin has been found in some studies to be associated with higher-grade tumors. ${ }^{12,13}$

All 11 cases stained intensely positive for Galectin-3 (Figure 8). Diffuse-strong expression of galectin-3 as seen in these cases has been found in recent studies to be an indicator of tumor progression and metastasis in pancreatic carcinomas. ${ }^{14}$

Positivity for CD31 was evident focally in the areas of micropapillary pattern in only one case out of the 11.

All the internal and external controls stained appropriately.

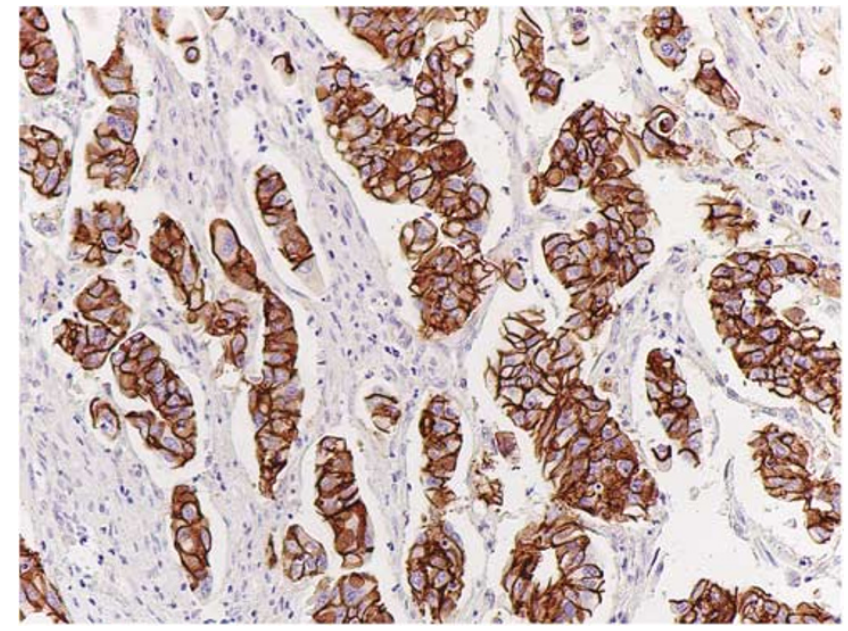

Figure 7 Cytoplasmic and membranous immunostaining by $\mathrm{E}$ cadherin ( $9 / 11$ cases).

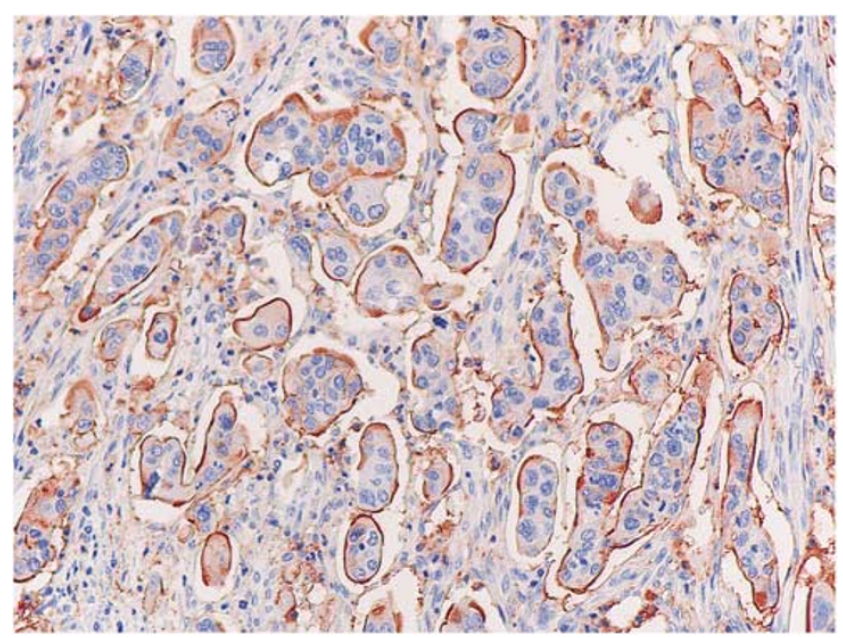

Figure 6 Predominant MUC 1 immunostaining in the stromafacing surface of the cell clusters.

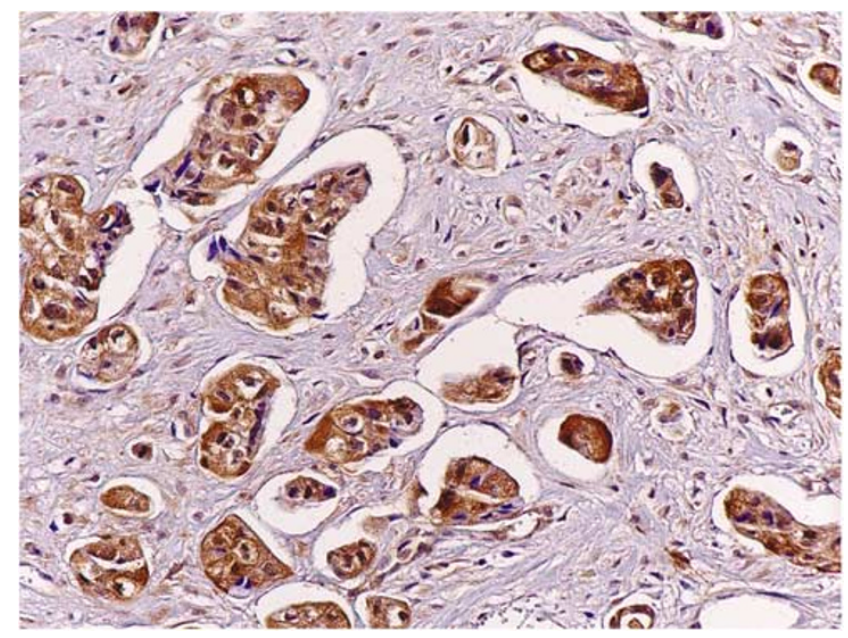

Figure 8 Diffuse strong immunostaining by galectin-3 (11/11 cases). 
Table 3 IHC stain pattern of pancreatic adenocarcinoma cases with MP component

\begin{tabular}{lcccc}
\hline Case \# & $\begin{array}{c}\text { Stroma-facing } \\
\text { MUC 1 } \\
\text { staining }\end{array}$ & $\begin{array}{c}\text { Abnormal } \\
\text { E-cadherin } \\
\text { staining }\end{array}$ & Galectin-3 & CD31 \\
\hline 1 & N/A & N/A & N/A & N/A \\
2 & + & + , cytoplasmic & +++ & 0 \\
3 & + & + , cytoplasmic & +++ & + \\
4 & + & + , cytoplasmic & +++ & 0 \\
5 & + &,+ cytoplasmic & +++ & 0 \\
6 & + & + , cytoplasmic & +++ & 0 \\
7 & + & + cytoplasmic & +++ & 0 \\
8 & + & 0 & +++ & 0
\end{tabular}

N/A = not applicable.

Table 4 IHC stain pattern of periampullary adenocarcinoma cases with MP component

\begin{tabular}{lcccc}
\hline Case \# & $\begin{array}{c}\text { Stroma-facing } \\
\text { MUC } 1\end{array}$ & $\begin{array}{c}\text { Abnormal } \\
\text { E-cadherin }\end{array}$ & Galectin-3 & CD31 \\
\hline 1 & N/A & N/A & N/A & N/A \\
2 & N/A & N/A & N/A & N/A \\
3 & + & 0 & +++ & 0 \\
4 & N/A & N/A & N/A & N/A \\
5 & + &,+ cytoplasmic & +++ & 0 \\
6 & + & + cytoplasmic & +++ & 0 \\
7 & N/A & N/A & N/A & N/A \\
8 & + & +, cytoplasmic & +++ & 0 \\
\hline
\end{tabular}

N/A $=$ not applicable.

Immunohistochemical findings are summarized in Tables 3 and 4 .

\section{Electron Microscopy}

Ultrastructural examination of one of the cases showed the presence of a large number of microvilli at the surface of the cells facing the stroma (Figure 9), as observed in micropapillary tumors of other organs. ${ }^{15}$

\section{Stage and Grade}

In all, 11 of 15 cases $(73 \%)$ in which the lymph node status was available had lymph node metastasis, similar to the $55 \%$ of the non-micropapillary cases in the same database $(P=0.267)$. Other sites of metastasis that were recorded clinically subsequent to the resections included the liver $(n=3)$, and the lung $(n=1)$.

In terms of grading, as a nonglandular growth pattern, invasive micropapillary carcinoma would qualify as Grade 3 in the recently proposed grading scheme of pancreatic adenocarcinoma, ${ }^{16}$ and the survival analysis below supports this impression.

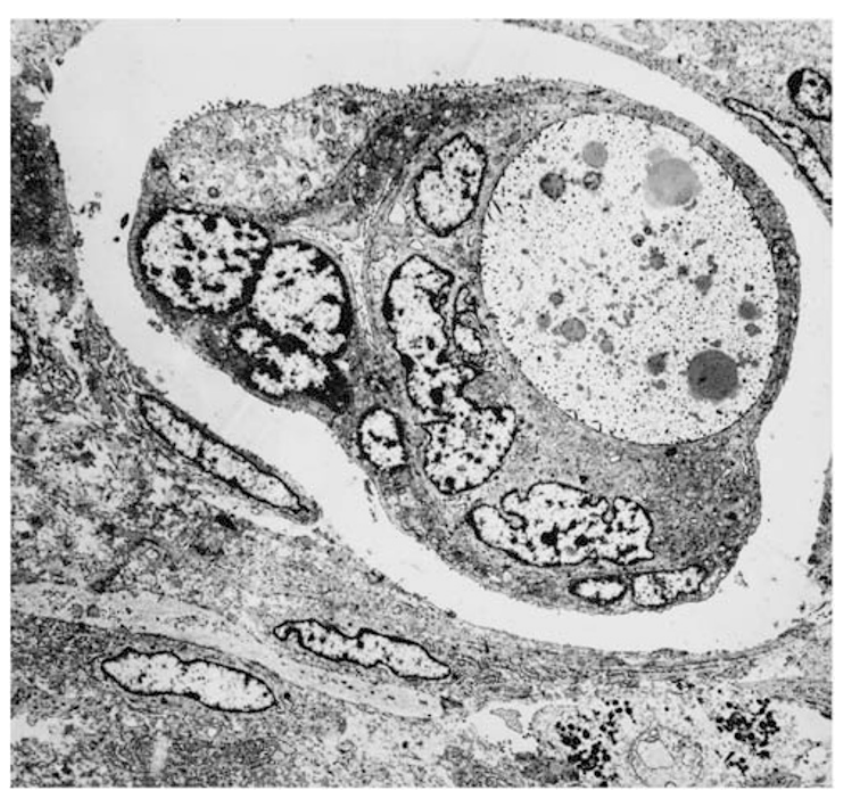

Figure 9 Electron microscopy, showing large number of microvilli at the stroma-facing surface.

When graded by the WHO/Kloppel system, 13 out of the 16 cases fell into the G3 category (average tumor grade score 2.71). ${ }^{17}$

The number of cases was too small to evaluate the correlation between the proportion of micropapillary pattern and biologic behavior.

\section{Clinical Outcome}

Adequate follow-up data were available for 14 cases. Follow-up ranged from 2 to 70 months. The median survival was 8 months (mean 17 months; confidence interval 6-10 months). At last follow-up, only two patients remained alive. Although the number of cases was small, the survival of the patients appeared to be slightly worse than that of average ordinary ductal carcinoma of the pancreas in the same database (mean 20 months, median 13 months) $(P=0.342)$. Also, this survival data correlate with the Grade 3 (poorly differentiated) category in the recently proposed grading scheme, which was found to have a median survival of 17 months (confidence interval =9-25) for Grade 1, 14 months (confidence interval $=12-16$ ) for Grade 2 and 7 months (confidence Interval $=5-9$ ) for Grade $3 .{ }^{16}$ (Figure 10).

\section{Discussion}

Invasive Micropapillary Carcinomas Occur in the Ampullo-Pancreatobiliary Region and are Similar to those of Other Organs

This study documents that invasive micropapillary carcinoma, a distinctive tumor type originally 


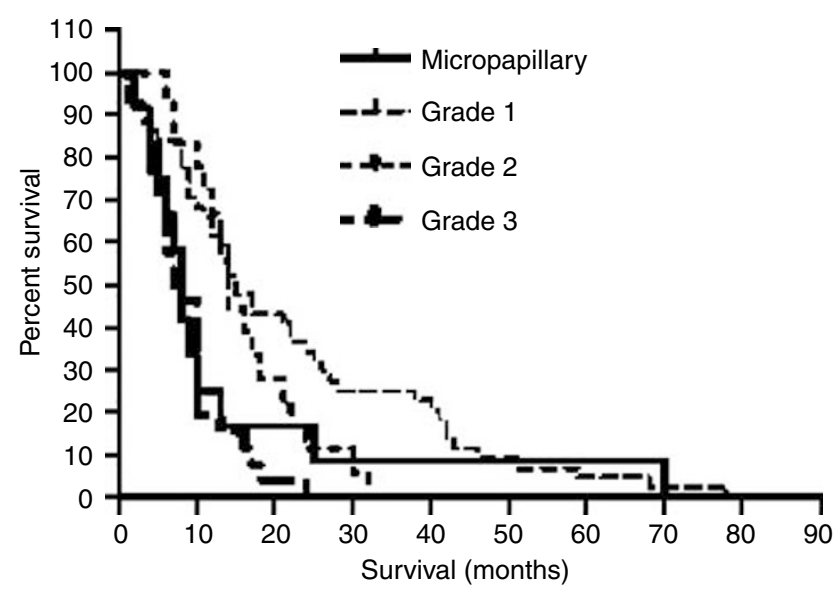

Figure 10 Invasive micropapillary carcinoma survival compared to Grades 1, 2 and 3 of ordinary pancreatic carcinoma. Invasive micropapillary carcinoma survival data would place it in the Grade 3 (poorly differentiated) category.

described in the breast, urinary bladder, lung and salivary glands, ${ }^{16}$ does arise in the ampullopancreatobiliary region as well. In the breast, it was reported to occur in $6 \%$ of the cases. ${ }^{9}$ In the ampullo-pancreatobiliary region, micropapillary pattern is encountered in $4.1 \%$ of the carcinomas. It appears to be more common in the ampullary region $(1 \%)$ than in the pancreas $(3 \%)$. Tumors composed almost exclusively of this pattern ( $>80 \%$ of the tumor), however, are very rare $(3 \%$ in the ampulla and $1 \%$ in the pancreas). It should be kept in mind that metastatic carcinomas with micropapillary pattern also occur in this region, and the authors have encountered an example of urothelial micropapillary carcinoma metastatic to pancreas presenting like a primary carcinoma. ${ }^{18}$ Also, angioinvasive components of otherwise ordinary adenocarcinomas may have a micropapillary appearance, which should be differentiated from invasive micropapillary carcinoma. On the other hand, the mechanisms of cell detachment from the vascular endothelium that leads to the 'micropapillary' clusters in the vessels may be very similar to that of the invasive micropapillary carcinoma, and furthermore, their biologic significance may also be similar. The expression pattern of MUC 1 supports this possibility. Both the micropapillary clusters in angioinvasive components of ordinary carcinomas as well as invasive micropapillary carcinoma itself show MUC 1 immunoexpression in the stromafacing (or endothelium-facing, in the case of angioinvasive clusters) surfaces of the cells, which is in contrast with the strictly luminal expression seen in the ordinary tubular infiltration in the stroma. This phenomenon was observed in all 11 cases in which tissue was available for staining. As documented recently, ${ }^{10}$ MUC 1 , which has an important physiologic role in the maintenance of lumina and an inhibitory role in tumor cell-stroma interaction, may be an important factor in the detachment of the cells from the stroma, and thus responsible for the micropapillary morphology as well as easier spread of the cells by dissection of the stroma.

\section{The Clinical Presentation of Invasive Micropapillary Carcinoma in this Region is Similar to that of Pancreaticobiliary Type Adenocarcinoma}

Invasive micropapillary carcinomas of the ampullopancreatobiliary region do not appear to differ significantly from the conventional (pancreatobiliary type, pancreatic ductal) adenocarcinomas, except that they may be slightly more common in males (male:female ratio $=10: 6 ; 63 \%$ in males, vs almost equal-or slightly female predominantdistribution of pancreatobiliary adenocarcinomas). The mean age is 69 years as opposed to 63 years in ordinary ductal adenocarcinomas. The propensity for lymph node metastasis characteristic of micropapillary carcinomas of other organs, on the other hand, is difficult to verify in this region, since the carcinomas in this area already show significant lymphotropism. In all, 11 of $15(73 \%)$ cases in this study had lymph node metastasis, which is similar to the incidence of lymph node metastasis of micropapillary carcinomas in other organs, ${ }^{9}$ and also similar to that of the conventional adenocarcinomas of this site $(55 \%)$.

\section{Invasive Micropapillary Carcinoma of this Region is a High-Grade Adenocarcinoma}

It is possible that invasive micropapillary carcinoma of this region were previously categorized under the heading of 'poorly differentiated adenocarcinomas (not otherwise specified)'. Indeed, the clinical, morphologic and immunophenotypic findings in this study indicate that these tumors are high-grade carcinomas.

Although the number of cases are too small to make a definitive statement about the biologic behavior of invasive micropapillary carcinoma of ampullo-pancreatobiliary region, the median survival of the patients in this study (8 months) appears to parallel that of the Grade 3 (poorly differentiated) category in the recently proposed grading scheme, which showed 22, 14 and 8 months median survival for Grades 1, 2 and 3 categories, respectively. This is in accordance with the principles of this grading scheme, which regards nonglandular patterns (which invasive micropapillary carcinoma would qualify for) as Grade $3 .{ }^{16}$

Immunohistochemical findings also support the high-grade nature of these carcinomas. Galectin-3 expression was diffuse and strong in all 11 cases studied. Galectins are a family of $\beta$-galactosidebinding protein that have been proposed to regulate cell growth, to mediate cell adhesion and to influence apoptosis. However, galectin-3 appears 
to be involved in cancer cell development. ${ }^{19}$ High expression of galectin-3 has been found to be an indicator of tumor progression and metastasis in pancreatic carcinomas, although there is conflicting data in the literature regarding this issue. ${ }^{19,20}$

Similarly, cytoplasmic relocalization of E-cadherin, which was found to be significantly more common in poorly differentiated carcinomas of the pancreas, ${ }^{12,13,21}$ was detected in nine of 11 cases in this study. E-cadherin-catenin complex is a major indicator of intercellular adhesion and epithelial integration maintenance. The abnormal structure and dysfunction of one or several molecules in E-cadherin-catenin complex correlates with tumorigenesis, poor differentiation, metastasis and aggressive behavior. ${ }^{12}$

\section{Invasive Micropapillary Carcinoma of the Ampullo-Pancreatobiliary Region are Often Associated with Tumor-Infiltrating Neutrophils, a Finding that is Uncommon in IMPCas of Other Sites as well as Carcinomas of this Area}

One peculiar finding in invasive micropapillary carcinoma of ampullo-pancreatobiliary region noted in this study is the presence of tumor-infiltrating neutrophils. This was present in nine of 16 cases, and was prominent especially in micropapillary-predominant cases. Neutrophils were abundant and could be identified both within the carcinoma cells ('cannibalism') as well as in the stroma adjacent to the tumor cells. Focal microabscesses in the vicinity of the tumor cell clusters could also be seen. No foreignbody giant cell reaction, suture granulomas, xanthoma cells, chronic inflammation, granulation tissue or scarring were identified in the vicinity of the tumors; therefore, the neutrophilic infiltrates could not be attributed to prior instrumentation, biopsy, ulceration or perforation. Furthermore, the neutrophils showed striking tumoral-centric distribution, decreasing in numbers away from the tumor cells. It is difficult to determine the meaning of this peculiar phenomenon. In fact, it is difficult to determine whether this could be a 'chicken and egg' phenomenon, and whether neutrophils are recruited preferentially to micropapillary carcinomas, or neutrophilic infiltrates participate in the development of micropapillary pattern. Or, some unexplained factors might be responsible for both. Regardless, this phenomenon is not reported in micropapillary carcinomas of other organs, nor was it common in ordinary carcinomas of this site. We believe that this may be an interesting topic for investigation.

\section{Conclusion}

Although rare, micropapillary pattern can be encountered in the ampullo-pancreaticobiliary region and appears to be more common in the ampulla than in pancreas. Invasive micropapillary carcinoma of this region appears to be slightly more common in males. Biologically and prognostically they appear to be high-grade (Grade 3 of 3 ) carcinomas. The preponderance of tumor-infiltrating neutrophils in invasive micropapillary carcinoma of this region is an interesting phenomenon that warrants further analysis.

\section{Acknowledgements}

This study was supported in part by the National Cancer Institute Specialized Program in Research Excellence (SPORE), CA101936 in pancreatic cancer.

\section{References}

1 Amin MB, Ro JY, el-Sharkawy T, et al. Micropapillary variant of transitional cell carcinoma of the urinary bladder. Histologic pattern resembling ovarian papillary serous carcinoma. Am J Surg Pathol 1994;18: 1224-1232.

2 Johansson SL, Borghede G, Holmang S. Micropapillary bladder carcinoma: a clinicopathological study of 20 cases. J Urol 1999;161:1798-1802.

3 Amin MB, Tamboli P, Merchant SH, et al. Micropapillary component in lung adenocarcinoma: a distinctive histologic feature with possible prognostic significance. Am J Surg Pathol 2002;26:358-364.

4 Nagao T, Gaffey TA, Visscher DW, et al. Invasive micropapillary salivary duct carcinoma: a distinct histologic variant with biologic significance. Am J Surg Pathol 2004;28:319-326.

5 Burks RT, Sherman ME, Kurman RJ. Micropapillary serous carcinoma of the ovary. A distinctive low-grade carcinoma related to serous borderline tumors. Am J Surg Pathol 1996;20:1319-1330.

6 Eichhorn JH, Bell DA, Young RH, et al. Ovarian serous borderline tumors with micropapillary and cribriform patterns: a study of 40 cases and comparison with 44 cases without these patterns. Am J Surg Pathol 1999;23:397-409.

7 Nassar H. Carcinomas with micropapillary morphology: clinical significance and current concepts. Adv Anat Pathol 2004;11:297-303.

8 Maranchie JK, Bouyounes BT, Zhang PL, et al. Clinical and pathological characteristics of micropapillary transitional cell carcinoma: a highly aggressive variant. J Urol 2000;163:748-751.

9 Nassar H, Wallis T, Andea A, et al. Clinicopathologic analysis of invasive micropapillary differentiation in breast carcinoma. Mod Pathol 2001;14:836-841.

10 Nassar H, Pansare V, Zhang $\mathrm{H}$, et al. Pathogenesis of invasive micropapillary carcinoma: role of MUC1 glycoprotein. Mod Pathol 2004;17:1045-1050.

11 Pernick NL, Sarkar FH, Philip PA, et al. Clinicopathologic analysis of pancreatic adenocarcinoma in African Americans and Caucasians. Pancreas 2003;26:28-32.

$12 \mathrm{Li} \mathrm{YJ,} \mathrm{Ji} \mathrm{XR.} \mathrm{Relationship} \mathrm{between} \mathrm{expression} \mathrm{of} \mathrm{E-}$ cadherin-catenin complex and clinicopathologic characteristics of pancreatic cancer. World J Gastroenterol 2003;9:368-372.

13 Karayiannakis AJ, Syrigos KN, Chatzigianni E, et al. Aberrant E-cadherin expression associated with loss of 
differentiation and advanced stage in human pancreatic cancer. Anticancer Res 1998;18:4177-4180.

14 Schaffert C, Pour PM, Chaney WG. Localization of galectin-3 in normal and diseased pancreatic tissue. Int J Pancreatol 1998;23:1-9.

15 Luna-More S, Gonzalez B, Acedo C, et al. Invasive micropapillary carcinoma of the breast. A new special type of invasive mammary carcinoma. Pathol Res Pract 1994;190:668-674.

16 Adsay NV, Basturk O, Bonnett M, et al. A proposal for a new and more practical grading scheme for pancreatic ductal adenocarcinoma. Am J Surg Pathol 2005;29: 724-733.

17 Luttges J, Schemm S, Vogel I, et al. The grade of pancreatic ductal carcinoma is an independent prognostic factor and is superior to the immunohistochemical assessment of proliferation. J Pathol 2000; 191:154-161.
18 Adsay NV, Andea A, Basturk O, et al. Secondary tumors of the pancreas: an analysis of a surgical and autopsy database and review of the literature. Virchows Arch 2004;444:527-535.

19 Berberat PO, Friess H, Wang L, et al. Comparative analysis of galectins in primary tumors and tumor metastasis in human pancreatic cancer. J Histochem Cytochem 2001;49:539-549.

20 Shimamura T, Sakamoto M, Ino Y, et al. Clinicopathological significance of galectin-3 expression in ductal adenocarcinoma of the pancreas. Clin Cancer Res 2002;8:2570-2575.

21 Yonemasu H, Takashima M, Nishiyama KI, et al. Phenotypical characteristics of undifferentiated carcinoma of the pancreas: a comparison with pancreatic ductal adenocarcinoma and relevance of E-cadherin alpha catenin and beta catenin expression. Oncol Rep 2001;8:745-752. 